\title{
Color Channel Normalization and Fuzzy Filter based Image Fusion in Discrete Wavelet Domain
}

\author{
Mandeep Kaur \\ M. Tech Student \\ Amritsar College of Engineering and Technology \\ Amritsar
}

\author{
Aarti \\ Assistant Professor \\ Amritsar College of Engineering and Technology \\ Amritsar
}

\begin{abstract}
In Multi-focus image fusion has appeared as a significant subject in computer perspective and picture handling neighborhood because the visual contacts have a decreasing target range. Multi-focus picture fusion method have been commonly utilised in machine vision, targeting, object acceptance, medical imaging and military affairs. In nearly all past image fusion study, the origin images are thought as completely aligned. But still it has some issues which are neglected for image fusion. This paper proposes two level Discrete Wavelet Transformation (DWT). The proposed methodology has indicated really significant improvements around available ones.
\end{abstract}

\section{Keywords}

Image Fusion, 2DWT, Color Normalization, Noises, Fuzzy Filters.

\section{INTRODUCTION}

Image Fusion is a vital in picture processing. It is a class to pairing relevant knowledge from several photographs correct in to a solitary picture where in reality the resultant merged picture is more instructive image. The fused picture holds all of the important knowledge as distinction to data images. The fused picture might obtain all the information from supply images. With rapidly development in design, it happens to be conceivable to gain knowledge from multi-source photographs to make higher merged image. Brought on by picture mix is interchange picture that remaining places the utter most wonderful information and features of knowledge. Image fusion is really a of good use method of pairing the simple warning and multi-sensor photographs to boost the data. Passion behind image merge is always to become defined on knowledge from couple of photographs recall the finish aim to generate a visual that communicates just the of use information, clear, and understandable. Multi-focus picture fusion supplies a encouraging way to increase level of defocused photos by mixing numerous images of varied focuses right into a simple target one .Picture fusion can manage numerous information, complementary, redundant and from different sources or homogeneous to acquire the best possible knowledge of the environment studied decision. Picture fusion is worried with the integration or assortment of multiple different images, based on different detectors, in to a single image which will be more ideal for visual belief or computer-processing jobs. Image fusion is the method of combining numerous resource photos from indicator network right into a single one, which has a more precise explanation of the scene, more educational and suitable for equally visual perception.

\subsection{Levels of image Fusion}

Image fusion practices mix knowledge from various sources collectively. The key target of image merge is to create fused result that gives the most detailed and trusted data possible. Fusion of multiple data sources together also produces a more proficient representation of the data. There are three various categories of image fusion.Pixel stage mix could be the mixture of the natural knowledge from numerous supply photographs into a single image. Function stage mix needs the removal of various crucial features from origin knowledge before functions are merged together. Decision stage mix mixes the outcomes from numerous methods to deliver your final merged choice.

\subsection{Image fusion practices}

In these practices great data from one of the provided images is merged together to create a resultant image whose quality is superior to some of the insight images.

Image fusion strategies can be categorized into two methods i.e.

\subsubsection{Spatial Domain Fusion Method}

Averaging, Brovery method and Crucial Element Examination (PCA) centered methods are spatial domain methods. Spatial domain picture mixture techniques are difficult and extended which are complex categorized to be executing on real-time programs. Spatial domain techniques create distortion in the mixed image and that case could be handle by change domain technique. In spatial domain practices, we straightly cope with the pixel specified outcome. In spatial domain techniques, we immediately cost of a image. The pixel costs are improved to attain the cope with the pixel price of pictures. The pixel prices are altered to get the desire outcome. In frequency domain methods the pixel prices is first moved into domain methods by fusion methods. Next picture is improved by changing frequency element of a picture. Picture merge is used in most area wherever pictures have to be analyzed. For instance, microscopic imaging, medical picture analysis, analysis of pictures from satellite, computer perspective and battlefield checking.
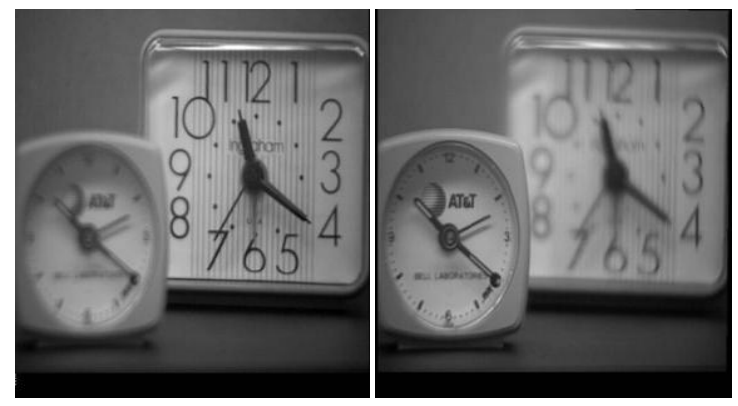

Fig 1: Left and Right Blurred 


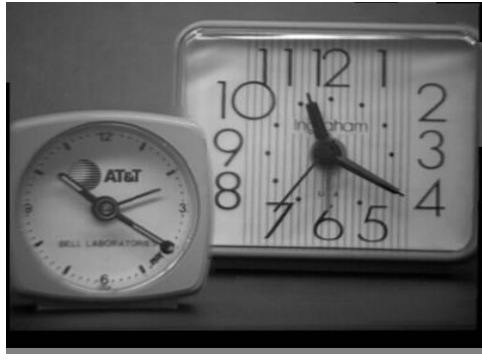

Fig 2: Fused image

\subsubsection{Transform domain method}

In modify domain strategy, original picture is transformed in to volume domain. The combination technique for instance DWT and DCT comes below modify domain technique.

\subsubsection{Discrete Cosine Transform (DCT)}

Spatial domain picture synthesis practices are difficult and time intensive which are difficult to be done on real-time photographs. Furthermore, once the origin photos are numbered in combined Photographic professional class format or when the merged picture is likely to be preserved in JPEG structure, then the synthesis practices which are used in DCT domain will soon be really efficient. To do the JPEG coding, a picture is first splitted in to prevents of $8 \times 8$ pixels. The Distinct Cosine Transform (DCT) is then done on every segment.

\subsubsection{Discrete Wavelet Transform (DWT)}

The Wavelet convert separate picture into low-low, low-high, high-low, high-high spatial volume groups of various volume bands. The LL group has calculate coefficients .LH groups contain also aspect coefficients. HL groups contain straight points of interest coefficients. HH contains straight aspect coefficients. The calculate and total coefficients of equally pictures are joined together using Mix rule. The merged picture could possibly be purchased by taking the inverse distinct wavelet transform. The first key part reveals much of the residual of the huge difference as can fairly be expected. To initialize with key part is sent to be alongside path with the very best variance. Another key part is forced to lay while in the subspace perpendicular in the first. Inside that Subspace, that part stresses the road of best variance. The 3rd key part is taken the utter most exorbitant deviation path while in the subspace perpendicular to the initial two and thus on.

\section{IMAGE NOISES}

\subsection{Gaussian Noise}

Removing with greater standard deviations suppresses noise, but additionally blurs the image. Gaussian noise is mathematical noise utilizing a possibility density purpose (PDF) similar compared to that in the standard submitting, that will be also known as the Gaussian is submitting. In many other phrases, the prices the way the noise generally tend to be
Gaussian-distributed.

\subsection{Salt-and-Pepper Noise}

Impulsive" sound is may also be named as spike noise. A picture comprising salt-and-pepper noise may get black pixels in brilliant parts and bright pixels in dark regions.

\subsection{Shot Noise}

The principal noise in the deeper areas of a picture from a picture indicator is usually that brought on by mathematical quantum changes, that's, deviation in the amount of photons thought at a provided coverage level. That sound is recognized as photon shot noise.

\section{FILTER}

A brand new algorithm is presented that could remove behavioural instinct noise by corrupted images while conserving details. The algorithm is determined by unclear behavioural instinct detection alongside unclear disturbance termination techniques. Experimental outcomes show that the algorithm can giving considerable improvement over several printed techniques regarding equally really subjective and function evaluations. Eliminating behavioural instinct noise there are numerous linear alongside low linear filtering techniques.

\subsection{Fuzzy Filter}

This fuzzy filter is a very useful kind of filter. Unclear filtration is use for removing the combined noises from digital images.

\subsection{Gaussian filter}

The Gaussian filtration is in certain methods a bargain between these filters - its load stays good but reduces smoothly in both spatial and volume domains

\subsection{Mean filter}

We are able to use linear filter to eliminate particular forms of sound. Specific filters, as for example Gaussian filters are appropriate. Like an averaging filtering is helpful for eliminating wheat noise from the photograph. Because each pixel gets collection to the typical of the pixels in its neighbor space, regional modifications brought on by grain are decreased. Therefore linear filter Algorithms are requested for picture processing.

\subsection{Median filters}

Median filtration is a nonlinear electronic filtering method, frequently employed to get rid of unwanted sound. That noise decrease just normal pre-processing stage to enhance the outcomes of later running (for example, edge recognition on an image). Median selection is extreamly commonly utilised in digital electronic since below certain problems, it preserves sides though eliminating noise. 


\section{METHODOLOGY}

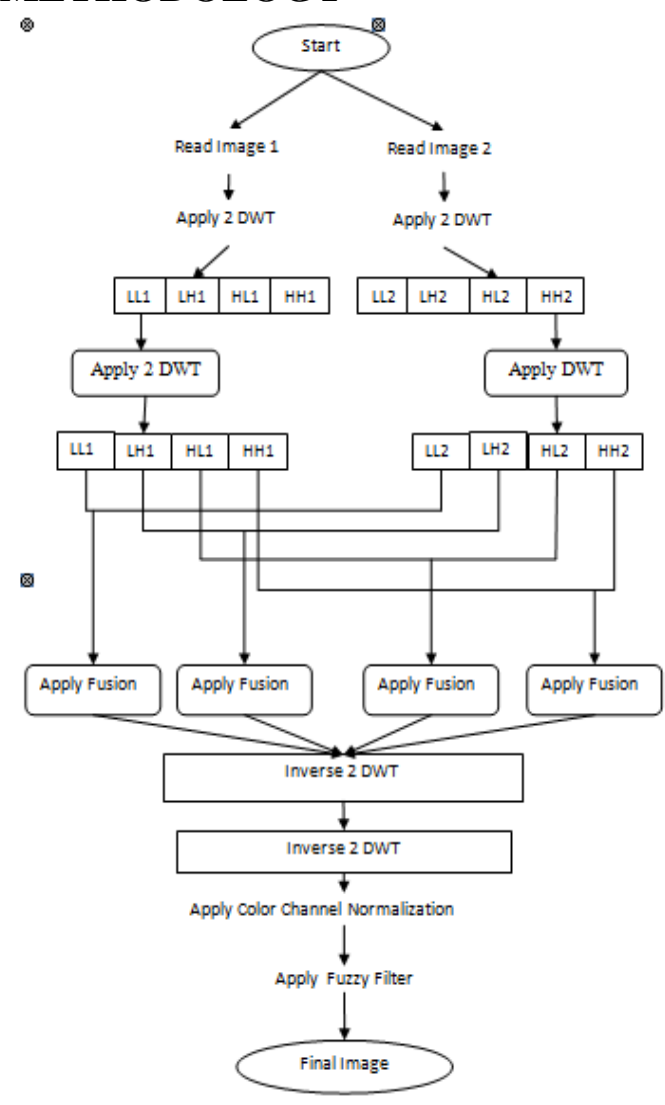

Fig 3: Methodology of the work

Step 1: Choose the images from pc storage in to pc program. Any provided digital image is displayed as an array size $M * N$ pixels.

Step 2: Use 2DWT technique on given images which will decompose the image into various levels.

Step 3: Again apply DWT technique which further decompose image into various levels.

Step 4: Then fuse the images to get final fused image.

Step 5: Apply inverse 2DWT Method.

Step 6: Again Apply inverse 2DWT Method.

Step 7: Apply Color Channel Normalization.

Step 8: Apply Fuzzy Filter.

Step 9: Final image which is more clear than input images

\section{RESULTS}

This paper has designed and implemented the proposed technique in MATLAB tool u2013a. The planned algorithm is very much better than the existing practices. A comparison is drawn between all the parameters and respective tables and figures show all the results

\subsection{PERFORMANCE ANALYSIS}

\subsubsection{Peak Signal to noise Ratio Evaluation}

PSNR have to be greatest; therefore key aim is to improve PSNR around possible. Table 1 has obviously revealed that PSNR has higher results in the case of the planned algorithm thus planned algorithm provides greater effects compared to the available practices.
Table 1. Peak Signal to Noise Relation Evaluation

\begin{tabular}{|c|c|c|}
\hline Images & $\begin{array}{c}\text { Existing } \\
\text { technique }\end{array}$ & $\begin{array}{c}\text { Proposed } \\
\text { technique }\end{array}$ \\
\hline 1 & 56.7210 & 90.0137 \\
\hline 2 & 52.8096 & 89.8052 \\
\hline 3 & 70.9534 & 88.9058 \\
\hline 4 & 64.6989 & 87.9922 \\
\hline 5 & 64.9143 & 85.8770 \\
\hline 6 & 66.8591 & 90.2799 \\
\hline 7 & 63.4195 & 85.7478 \\
\hline 8 & 61.4579 & 92.5754 \\
\hline 9 & 67.8597 & 94.3725 \\
\hline 10 & 59.9296 & 90.2738 \\
\hline
\end{tabular}

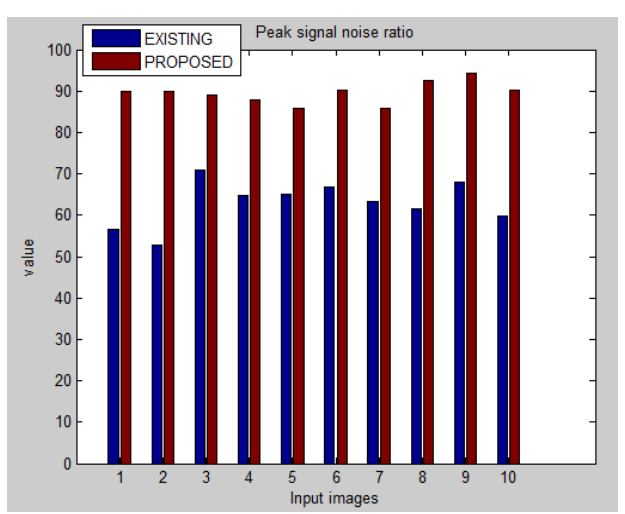

Fig 4: PSNR of Available Technique \& Planned Approach for different images

The above fig. 4 has indicates the quantized examination of the peak signal to noise ratio of various images using SWT by Existing Technique (Blue Color) and Discrete wavelet Transform by Planned Approach (Red Color). It has clearly shown from the table that there is escalation in PSNR value of images with the usage of planned method over available methods. This increase shows improvement in the objective quality of the images.

\subsubsection{Mean Square Error Evaluation}

As mean sq error has to be decreased which means planned algorithm is featuring the higher effects compared to the available practices as these values are less in all of the cases. Table 2 reveals the quantized examination of the mean square error.

Table2. Mean Square Error Evaluation

\begin{tabular}{|l|l|l|}
\hline Images & Existing technique & Proposed technique \\
\hline 1 & 94.848475 & 2.053040 \\
\hline 2 & 148.798690 & 2.102905 \\
\hline 3 & 18.425065 & 2.332340 \\
\hline
\end{tabular}




\begin{tabular}{|l|l|l|}
\hline 4 & 37.856015 & 2.591025 \\
\hline 5 & 36.928705 & 3.305445 \\
\hline 6 & 29.520780 & 1.991070 \\
\hline 7 & 43.863675 & 3.354985 \\
\hline 8 & 54.977245 & 1.528660 \\
\hline 9 & 26.308550 & 1.242955 \\
\hline 10 & 65.553920 & 58.023345 \\
\hline
\end{tabular}

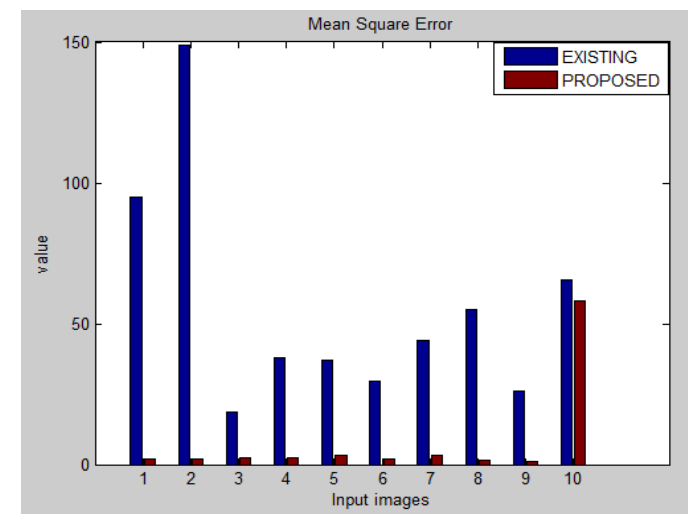

Fig 5: MSE of Current Technique \& Planned Approach for various images

The above fig. 5 describes the evaluation of the mean sq error of various pictures using SWT by current Technique (Blue color) and 2DWT by Planned Approach (Red Color). It has clearly shown from the table that there is fall in MSE value of pictures with the usage of planned strategies around other strategies in all pictures. This fall shows development in the target quality of the images

\subsubsection{Match Rate}

As match rate have to be maximized which means planned algorithm is featuring the better outcomes compared to the available practices as match rate is less in all of the cases. Table 3 reveals the quantized evaluation of the match rate.

Table3.Match Rate Evaluation

\begin{tabular}{|c|c|c|}
\hline Images & $\begin{array}{c}\text { Existing } \\
\text { technique }\end{array}$ & $\begin{array}{c}\text { Proposed } \\
\text { technique }\end{array}$ \\
\hline 1 & .8922 & .9855 \\
\hline 2 & .7741 & .9759 \\
\hline 3 & .9407 & .9871 \\
\hline 4 & .9788 & .9841 \\
\hline 5 & .9788 & .9826 \\
\hline 6 & .9534 & .9862 \\
\hline 7 & .9717 & .9853 \\
\hline 8 & .9466 & .9947 \\
\hline 9 & .9558 & .9866 \\
\hline 10 & .8225 & .9763 \\
\hline
\end{tabular}


Conference on.IEEE, 2013.

[7] Li, Shutao, Xudong Kang, and Jianwen Hu. "Image fusion with guided filtering."IEEE transactions on image processing: a publication of the IEEE Signal Processing Society 22.7 (2013): 2864-2875.

[8] Prakash, Om, RichaSrivastava, and AshishKhare "Biorthogonal wavelet transform based image fusion using absolute maximum fusion rule." Information \& Communication Technologies (ICT), 2013 IEEE Conference on.IEEE, 2013.

[9] Gupta, Vivek Kumar, AmitNeog, and S. K. Katiyar "Analysis of image fusion techniques over multispectral and microwave SAR images." Communications and Signal Processing (ICCSP), 2013 International Conference on.IEEE, 2013.

[10] Phamila, Y., and R. Amutha "Discrete Cosine Transform based fusion of multi-focus images for visual sensor networks." Signal Processing 95 (2014): 161-170.
[11] Parmar, K., \&Kher, R (2012, May). A comparative analysis of multimodality medical image fusion methods.In Modelling Symposium (AMS), 2012 Sixth Asia (pp. 93-97).IEEE.

[12] Tao, Ling, and Zhi-Yu Qian. "An improved medical image fusion algorithm based on wavelet transforms." Natural Computation (ICNC), 2011 Seventh International Conference on. Vol. 1. IEEE, 2011.

[13] Desale, Rajenda Pandit, and Sarita V. Verma. "Study and analysis of PCA, DCT \& DWT based image fusion techniques" IEEE International Conference on Signal Processing Image Processing \& Pattern Recognition (ICSIPR), Coimbatore, pp. 66-69, 7-8 Feb., 2013.

[14] Albuquerque, Hugo R., Tsang Ing Ren, and George DC Cavalcanti. "Image Fusion Combining Frequency Domain Techniques Based on Focus." Tools with Artificial Intelligence (ICTAI), 2012 IEEE 24th International Conference on. Vol. 1. IEEE, 2012. 